\title{
Upregulation of Soluble HLA-G in Chronic Left Ventricular Systolic Dysfunction
}

\author{
Line Lisbeth Olesen ${ }^{1}$ and Thomas Vauvert F. Hviid ${ }^{2}$ \\ ${ }^{1}$ Department of Cardiology, Zealand University Hospital (Roskilde), 10 Sygehusvej, 4000 Roskilde, Denmark \\ ${ }^{2}$ Department of Clinical Biochemistry, Centre for Immune Regulation and Reproductive Immunology (CIRRI), \\ Zealand University Hospital (Roskilde) and Department of Clinical Medicine, University of Copenhagen, \\ 10 Sygehusvej, 4000 Roskilde, Denmark \\ Correspondence should be addressed to Thomas Vauvert F. Hviid; tvh@regionsjaelland.dk
}

Received 19 May 2016; Revised 20 July 2016; Accepted 25 August 2016

Academic Editor: Enrico Fainardi

Copyright (c) 2016 L. L. Olesen and T. V. F. Hviid. This is an open access article distributed under the Creative Commons Attribution License, which permits unrestricted use, distribution, and reproduction in any medium, provided the original work is properly cited.

\begin{abstract}
Left ventricular systolic dysfunction (LVSD) defined by ejection fraction (EF) $<40 \%$ is common, serious but treatable, and correct diagnosis is the cornerstone of effective treatment. Biomarkers may help to diagnose LVSD and give insight into the pathophysiology. The immune system is activated in LVSD, and the immunomodulatory molecule human leukocyte antigen-G (HLA-G) may be involved. The primary aim was to measure soluble HLA-G (sHLA-G) in the blood in different stages of LVSD $(<30 \%$ and $30-40 \%)$, in the midrange $\mathrm{EF} 40-50 \%$, and in preserved $\mathrm{EF} \geq 50 \%$ and to validate sHLA-G as a LVSD biomarker. The secondary aim was to examine associations between HLA-G gene polymorphisms influencing expression levels and LVSD. The 260 study participants were $\geq 75$ years old, many with risk factors for heart disease or with known heart disease. Soluble HLA-G was significantly and uniformly higher in the groups with $\mathrm{EF}<50 \%(<30,30-40$, and $40-50 \%)$ compared to EF $>50 \%(p<0.0001)$. $\mathrm{N}$-terminal fragment-pro-B-type natriuretic peptide (NT-proBNP) and uric acid values were inversely related to EF. According to Receiver Operating Characteristic (ROC) curves NT-proBNP outperformed both sHLA-G and uric acid as biomarkers of LVSD. Soluble HLA-G in blood plasma was elevated in LVSD regardless of EF. A novel finding was that a combined $14 \mathrm{bp}$ ins-del/ +3142 SNP HLA-G haplotype was associated with $\mathrm{EF}<40 \%$.
\end{abstract}

\section{Introduction}

The new European guidelines for heart failure define three groups based on ejection fraction (EF): a group with reduced $\mathrm{EF}<40 \%$ (left ventricular systolic dysfunction (LVSD)); a group in the grey zone with EF in the midrange $40-49 \%$; and a group with preserved $\mathrm{EF} \geq 50 \%$. It is concluded that patients in the grey zone probably have mild systolic dysfunction and the reason for creating a separate group is to stimulate research into characteristics, pathology, and treatment of this group of patients [1]. A problem related to the grey zone is an uncertain definition of LVSD, as shown and described in the echocardiographic study of the present study [2].

Left ventricular systolic dysfunction affects about $2 \%$ of the population in the western world, including many with unrecognized LVSD. It accumulates in the elderly population because LVSD is the final stage in most cardiac diseases, mostly caused by atherosclerosis in the coronary arteries [3]. The prognosis is grave, but treatment can delay progression and reduce morbidity and mortality. Screening for systolic heart failure in high risk populations should be considered because correct diagnosis is the cornerstone of effective treatment.

Echocardiography is the gold standard to diagnose LVSD, but access is limited, and referral to echocardiography requires a well-founded suspicion of LVSD [3]. Thus it is pivotal to look for new biomarkers, which might also give a better insight into the pathophysiology because LVSD is a complex disorder with hemodynamic, metabolic, neurohormonal, inflammatory, and immunological changes [4]. New biomarkers should be validated against established biomarkers. 
B-type natriuretic peptide (or brain natriuretic peptide (BNP)) and N-terminal fragment-proBNP (NT-proBNP) are well established as diagnostic and prognostic biomarkers in LVSD, and combination with ECG might increase the specificity and the ability to screen for LVSD in high-risk populations $[5,6]$. NT-proBNP synthesis is initiated by LVSD via neurohormonal activation and increased wall stress in the heart, and it is a hemodynamic cardiac marker. Uric acid is an old biomarker that may be ready for a renaissance [7].

Almost every disease and any injury to the body are accompanied by inflammation and activation of the immune system. The inflammatory system is complex and crucial for survival, but it is a double-edged sword. In LVSD, proliferation of monocytes and macrophages is observed. There is an increase in harmful oxygen-free radicals, primarily produced by xanthine oxidase; uric acid reflects xanthine oxidase activity. Proinflammatory cytokines with detrimental effects on myocardial function include tumour necrosis factor(TNF-) $\alpha$, interleukin-1, and interleukin-6 [4, 8-10]. These evoke a counterbalance reaction with increased production of anti-inflammatory interleukin-10 and HLA-G [11, 12].

This study aims to evaluate HLA-G as a new biomarker for LVSD. Human leukocyte antigen (HLA)-G is an HLA class Ib molecule with immunomodulatory, immunosuppressive, and tolerance-inducing functions [13]. It is well described in pregnancy protecting the fetus from an immune response from the mother. It is associated with a lower risk of rejection of a transplanted organ, [13-16], and, in cases of heart transplantations, myocardial expression of HLA-G has been significantly correlated with low risk of rejection [17]. In contrast, in pathological conditions, like infections and cancer, in which a vigorous and maintained immune response is desirable, the expression of HLA-G is detrimental. In cancer, it has deleterious escape-effects and the expression of HLA-G by the tumour cells seems to accelerate relapse [18]. The HLA-G protein has under normal conditions a very restricted expression pattern [13]. It is expressed during pregnancy by extravillous cytotrophoblast cells at the fetomaternal interface and is important for inducing maternal tolerance to the semiallogenic fetus $[19,20]$. Furthermore, HLA-G is expressed by certain monocytes, $T$ cells, and dendritic cells [21]. Four membrane-bound HLA-G isoforms and three soluble HLA-G isoforms generated by alternative splicing have been reported [13, 22]. Membrane-bound fulllength HLA-G1 can also be cleaved from the cell surface by metalloproteinases [23].

A single published study has indicated that soluble HLAG (sHLA-G) in plasma is upregulated in patients with systolic heart failure, compared to healthy controls, and independent of NYHA class, EF, and other biomarkers [24]. The study included only ten control subjects who were markedly younger than the participants in the present study. The current study compares sHLA-G with the state-of-the-art biomarker NT-proBNP and with uric acid, both independent biomarkers, in order to clarify if sHLA-G in blood plasma can be used as a biomarker for LVSD in a group of high-risk elderly persons. For the first time, two polymorphisms in the HLA-G gene are investigated in relation to EF. Several studies have indicated that these polymorphisms modulate HLA-G expression.

\section{Materials and Methods}

2.1. Patients and Samples. Individuals $\geq 75$ years old from the general population and from a heart failure clinic, with heart disease risk factors or with former or present cardiac disease, especially LVSD, as well as healthy persons, were invited to participate. Two hundred and sixty subjects were included in the study. All participants provided written informed consent and the study was carried out in accordance with the ethical standards of the Declaration of Helsinki and was approved by the local ethics committee of Region Zealand and the Danish Data Protection Agency. Baseline characteristics of the study subjects are shown in Table 1.

While resting in supine position at room temperature all of the 260 subjects had a blood sample taken. Blood samples were obtained as EDTA plasma and heparin plasma samples, whole blood (EDTA tubes), and serum, with rapid flow from a large antecubital vein using standard venipuncture techniques. For the plasma samples, subsequently, centrifugation was performed to obtain platelet-poor plasma. The plasma supernatant was separated and the aliquot was transferred to cryotubes. All blood aliquots were stored at $-80^{\circ} \mathrm{C}$ until analyzed.

Within the same hour a transthoracic echocardiography was performed by an experienced level 3 echocardiographer using General Electric Vingmed Vivid 7 or 9 and MJS probe 1.5-4.0 MHz and following guidelines from the Danish Society of Cardiology. Based on EF the study subjects were divided into four groups: (1) $\geq 50 \%$ (preserved EF, considered as normal), (2) midrange EF 40-50\%, (3) LVSD with EF of $30-40 \%$, and (4) LVSD with EF $<30 \%$. Presence of moderate or severe valvular dysfunction was registered.

2.2. ELISA for Full-Length sHLA-G (The sHLA-G1 and HLAG5 Isoforms). The level of sHLA-G1/HLA-G5 molecules in blood plasma samples was determined by a commercially available sandwich enzyme immunoassay (ELISA) (EXBIO, Praha, Czech Republic) according to the manufacturer's instructions. This ELISA specifically detects sHLA-G1 and HLA-G5 in a $\beta 2$-microglobulin- $(\beta 2 \mathrm{~m}-)$ associated form. Samples were analyzed in duplicate on two independent assay plates, always with the same calibrators and positive and negative controls on each plate, and the first set of samples was also reanalyzed as the last samples to secure the reproducibility and precision of the assay. Blood plasma samples were diluted $1+3$ with the provided Dilution Buffer (60 $\mu \mathrm{L}$ samples to $180 \mu \mathrm{L}$ Dilution Buffer). Samples were thawed and mixed thoroughly and $100 \mu \mathrm{L}$ of diluted plasma were loaded in duplicate onto microtiter plates precoated with the monoclonal antibody MEM-G/9 (anti-HLA-G1/G5). The plates were then incubated overnight at $4{ }^{\circ} \mathrm{C}$ (with no shaking). Following five washing steps with $350 \mu \mathrm{L}$ of the supplied washing buffer, $100 \mu \mathrm{L}$ of conjugate solution was added and the plates were incubated at room temperature (RT) for one hour. The conjugate solution consisted of monoclonal antihuman $\beta 2$-microglobulin antibody labeled with horseradish 


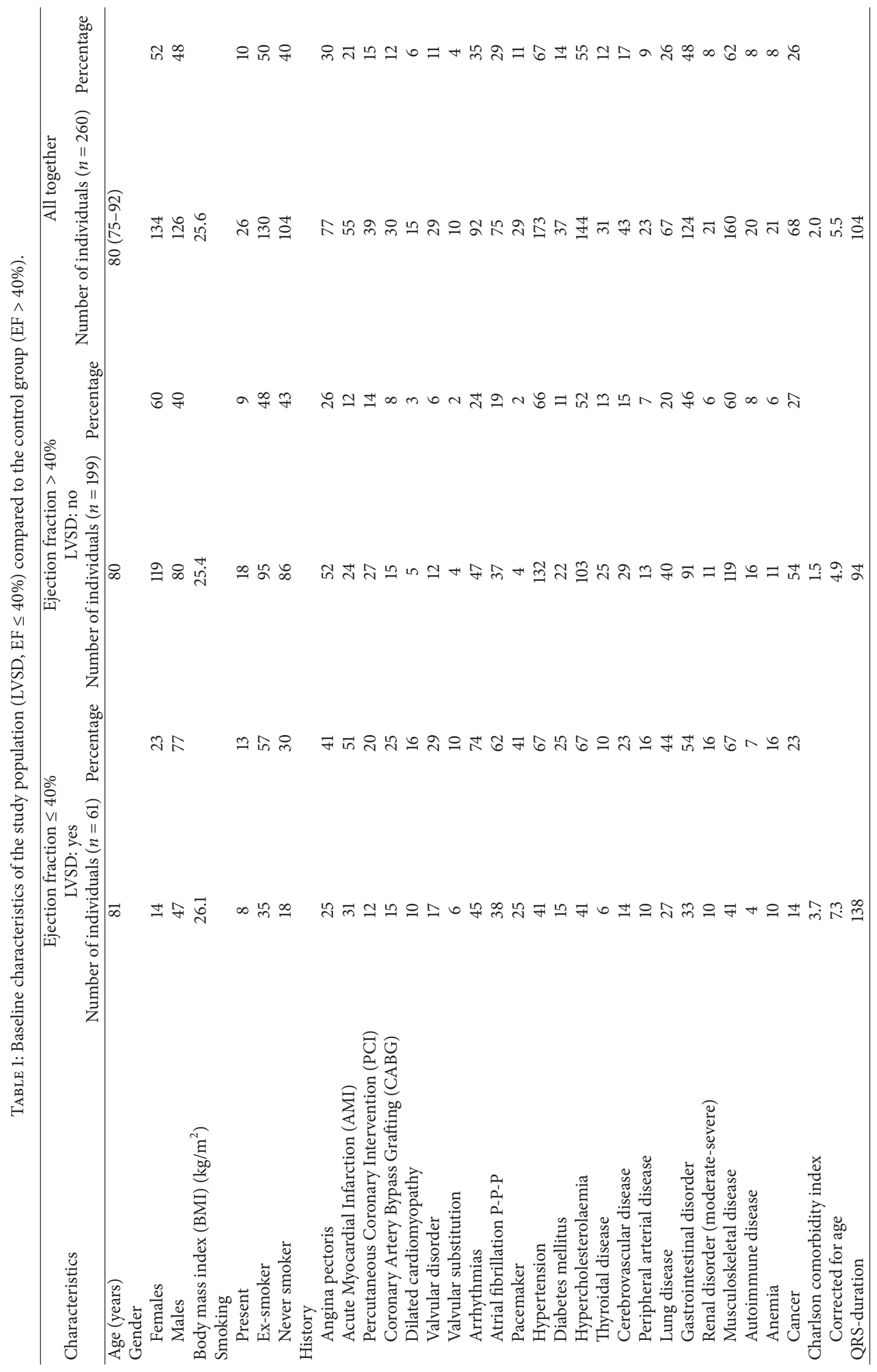




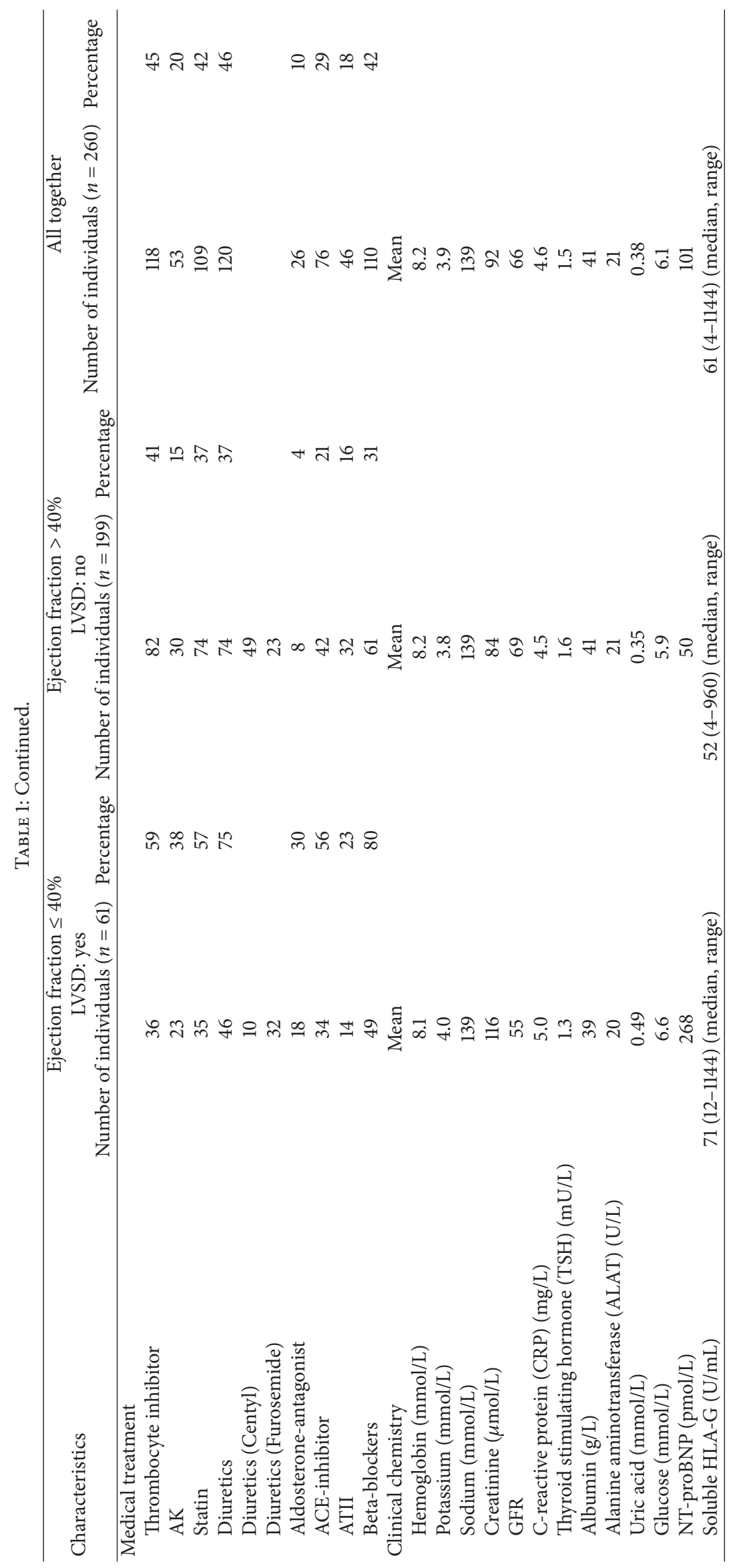


TABLE 2: The distribution of soluble HLA-G $(\mathrm{U} / \mathrm{mL})$, NT-proBNP (pmol/L), and uric acid $(\mathrm{mmol} / \mathrm{L})$ according to the left ventricular ejection fraction (median, range).

\begin{tabular}{lcccccc}
\hline Parameter & \multirow{2}{*}{ All study population } & \multirow{2}{*}{$n$} & \multicolumn{5}{c}{ Left ventricular ejection fraction } \\
& & & $>50 \%$ & $40 \%$ to $50 \%$ & $30 \%$ to $40 \%$ & $<30 \%$ \\
\hline Soluble HLA-G $(\mathrm{U} / \mathrm{mL})$ & $61(4-1144)$ & 259 & $44(4-960)$ & $90(11-758)$ & $71(12-1144)$ & $71(14-331)$ \\
NT-proBNP $(\mathrm{pmol} / \mathrm{L})$ & $35(4-1620)$ & 259 & $24(4-347)$ & $41(7-273)$ & $134(21-389)$ & $307(69-1620)$ \\
Uric acid $(\mathrm{mmol} / \mathrm{L})$ & $0.37(0.18-0.92)$ & 257 & $0.34(0.18-0.87)$ & $0.37(0.20-0.68)$ & $0.44(0.19-0.80)$ & $0.44(0.19-0.92)$ \\
\hline
\end{tabular}

peroxidase (HRP). After five additional washing steps, $100 \mu \mathrm{L}$ of substrate solution with tetramethylbenzidine $(\mathrm{TMB})$ were added to the plate, and the plate incubated once more at RT for 25 min with no shaking. Finally, $100 \mu \mathrm{L}$ of acidic stop solution were added to the plates. The plates were then analyzed using a microplate reader. The assay was performed in a BEP2000 ELISA robot instrument (Siemens Healthcare Diagnostics, Germany).

2.3. Analysis of NT-proBNP and Uric Acid. NT-proBNP was measured on the Elecsys 2010 system (Roche Diagnostics). The assay is an electrochemiluminescent sandwich immunoassay that uses two polyclonal antibodies directed at residues 1-21 and 39-50 of the NT-proBNP molecule. The CV\% of the assay is $3.2-2.4 \%$ from 20.7 to $585.5 \mathrm{pmol} / \mathrm{L}$ (175$4,962 \mathrm{ng} / \mathrm{L}$ ) with an analytical range of $0.6-4138.6 \mathrm{pmol} / \mathrm{L}$ (5$35,000 \mathrm{ng} / \mathrm{L}$ ). Plasma uric acid was measured on ARCHITECT ci8200 Integrated System (Abbott Diagnostics, North Chicago, IL, USA). One NT-proBNP test failed reducing the total number of study participants with a NT-proBNP test result to 259 .

2.4. Genotyping of the $14 \mathrm{bp}$ Insertion/Deletion Polymorphism (rs66554220) and the +3142 Single-Nucleotide Polymorphism (rs1063320) in the $3^{\prime}$-Untranslated Region of the HLA-G Gene. EDTA blood samples were carefully thawed and mixed thoroughly before processing. The DNA purification, using a Maxwell ${ }^{\circledR} 16$ DNA Purification Kit, was performed in accordance with the manufacturer's instructions, and genomic DNA was stored at $-20^{\circ} \mathrm{C}$ for further use. The real-time TaqMan PCR assay for genotyping of the HLA-G 14 bp insertion/deletion (ins/del) polymorphism in exon 8 (rs66554220) was performed using a LightCycler480 instrument (Roche Diagnostics, Switzerland) and performed as described by Djurisic et al. [25]. The genotyping of the +3142 SNP in the $3^{\prime}$ UTR of the HLA-G gene (rs1063320) was performed as described by Bortolotti et al. [26].

2.5. Statistical Analysis. Specific a priori hypotheses were formulated. Each variable was tested for Gaussian (normal) distribution. In cases of a normal distribution, parametric tests were used (one-way ANOVA and unpaired $t$-test). Else, nonparametric tests were used (Kruskal-Wallis test, MannWhitney $U$ test, and Jonckheere-Terpstra test). Receiver Operating Characteristic (ROC) curves were drawn for sHLA-G, proBNP, and uric acid. Charlson comorbidity index was calculated as described by Hall et al. [27]. Statistical analyses were made with the use of IBM SPSS version 22.0. Graphs were made in GraphPad Prism version 6 and IBM SPSS.

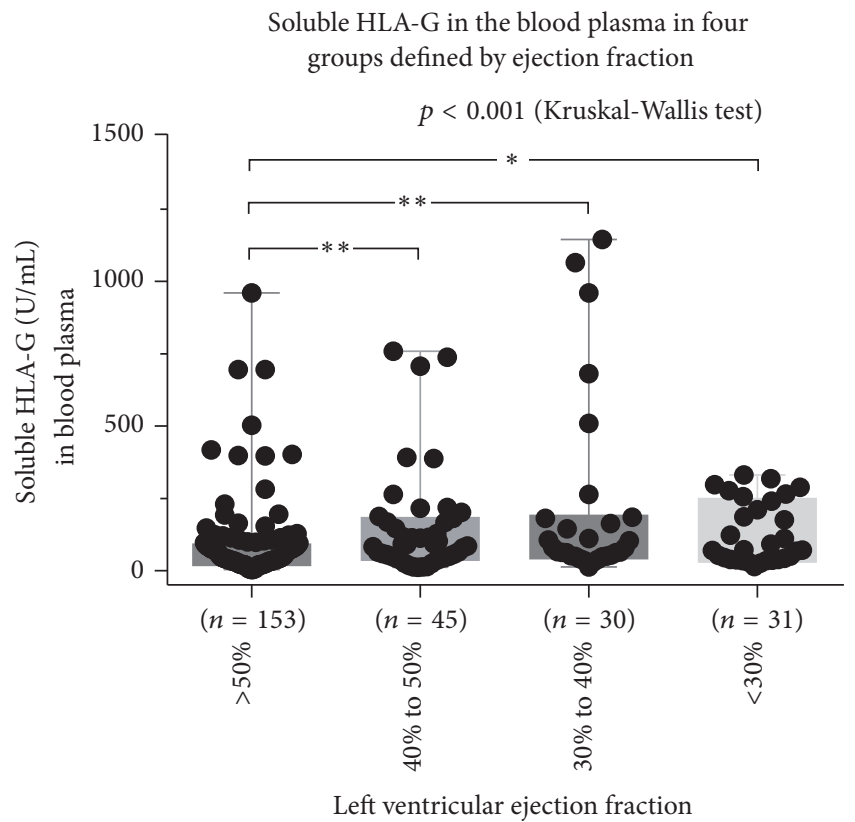

FIGURE 1: Soluble HLA-G in peripheral blood in relation to left ventricular ejection fraction (box and whiskers plot, min. to max., all points shown; $p<0.001$, Kruskal-Wallis test; Dunn's multiple comparisons test, ${ }^{*} p<0.05,{ }^{* *} p<0.01$ ) (one NT-proBNP test failed in the group with ejection fraction $>50 \%$ reducing the number to 153 ).

\section{Results}

3.1. Characteristics of the Study Group. Table 1 shows the baseline characteristics of the study group $(\mathrm{EF}<40 \%)$ versus the control group ( $\mathrm{EF} \geq 40 \%$ ). According to echocardiography there were $154(59.2 \%)$ subjects with $\mathrm{EF} \geq 50 \%, 106(40.8 \%)$ subjects with $\mathrm{EF}<50 \%$, 45 with $\mathrm{EF} 40-50 \%$, and 61 (23.5\%) with $\mathrm{EF}<40 \%$ (30 with EF $30-40 \%$ and 31 with $\mathrm{EF}<30 \%$ ); 55 subjects had mitral or aortic valvular dysfunction to some degree, no one had severe valvular disease, and 20 patients with valvular disease also had LVSD.

3.2. Soluble HLA-G Levels in Blood Plasma Are Associated with Heart Failure. Soluble HLA-G in the blood plasma was significantly and uniformly higher in the two LVSD groups with $\mathrm{EF}<30 \%$ and $\mathrm{EF} 30-40 \%$ and in the midrange group with EF 40-50\%, compared to the group with preserved $\mathrm{EF} \geq 50 \%(p<0.0001$, Kruskal-Wallis test (Figure 1 and Table 2)). The values of NT-proBNP and uric acid were increased with decreasing EF (Table 2). Furthermore, Receiver Operating Characteristic (ROC) curves showed that 


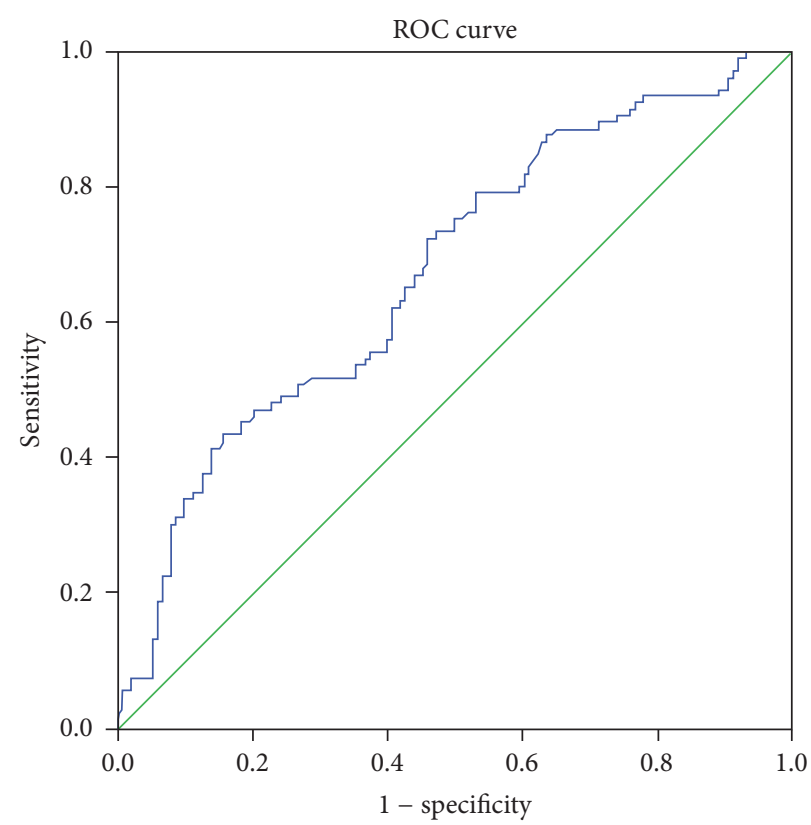

(a)

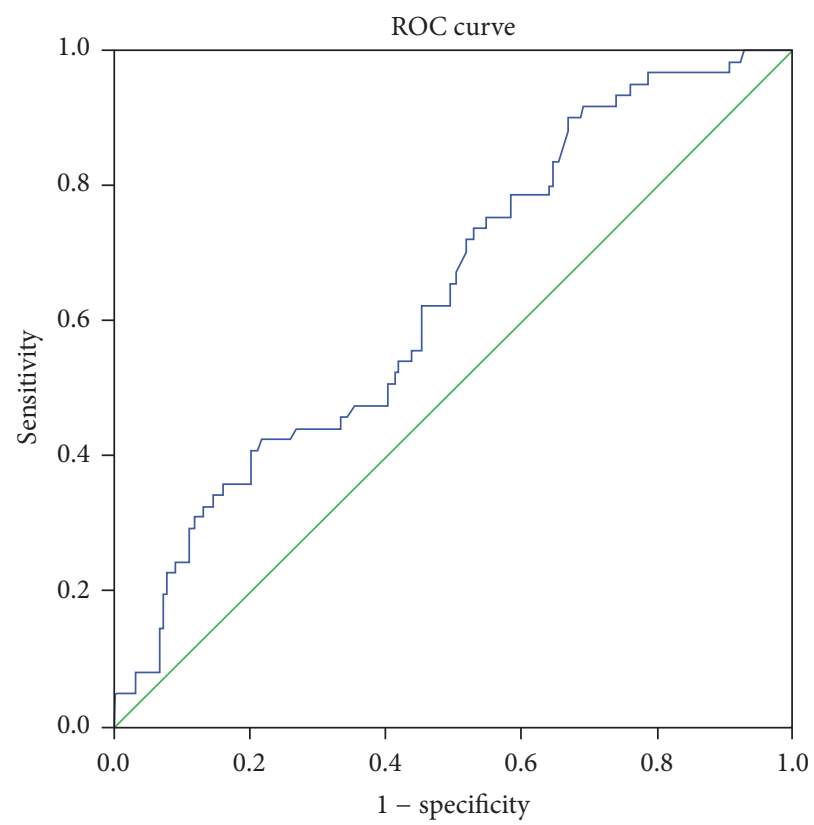

(b)

Figure 2: (a) Receiver Operating Characteristic (ROC) curve of soluble HLA-G with heart failure defined as ejection fraction $<50 \%$. Area under the curve is $0.676, p<0.001$. (b) Receiver Operating Characteristic (ROC) curve of soluble HLA-G with heart failure defined as ejection fraction $<40 \%$. Area under the curve is $0.639, p=0.001$.

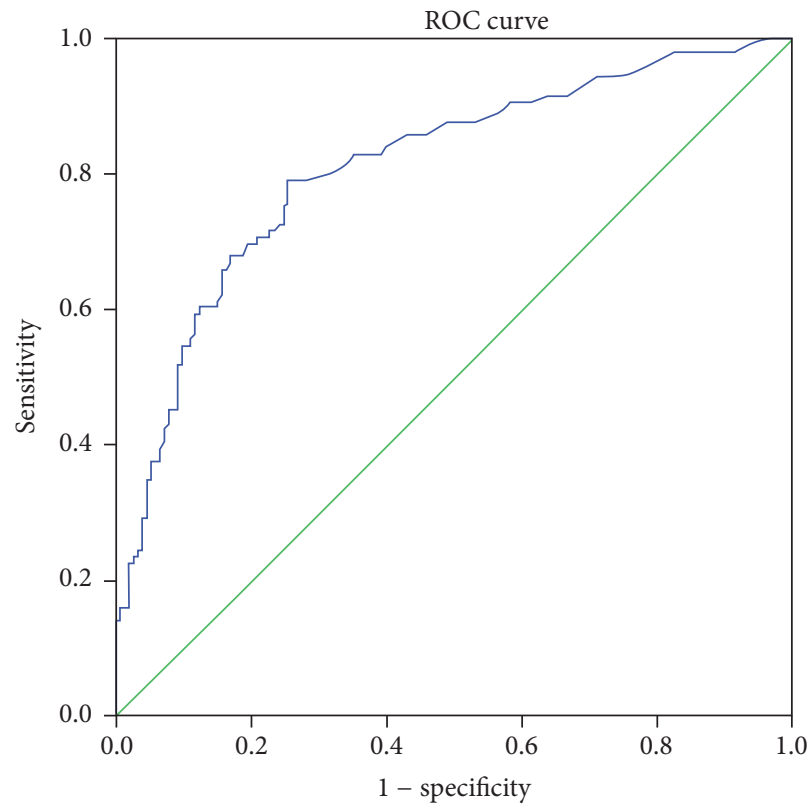

(a)

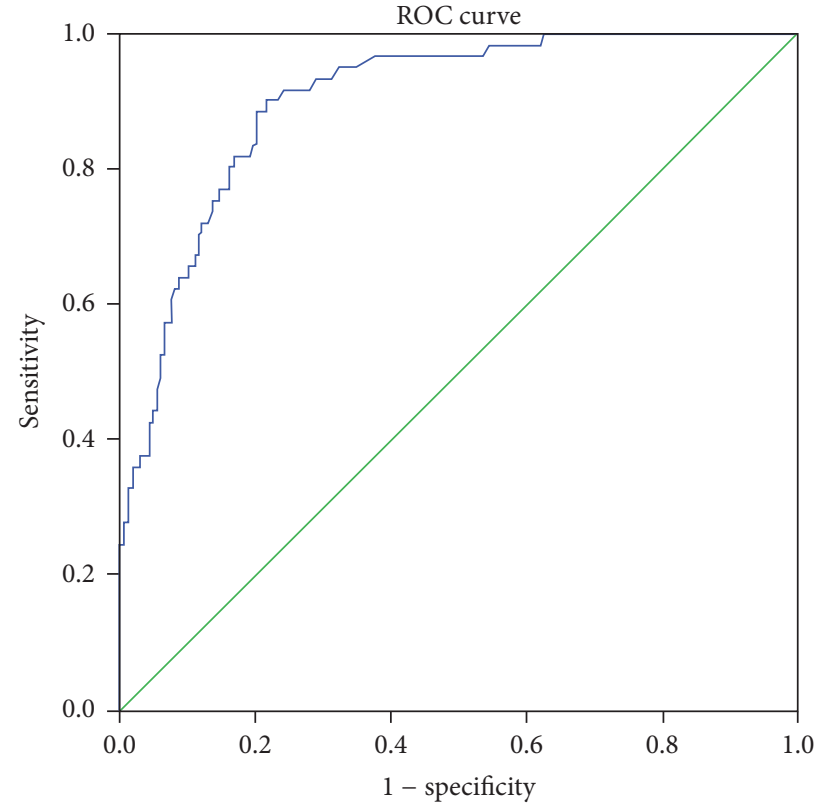

(b)

Figure 3: (a) Receiver Operating Characteristic (ROC) curve of NT-proBNP with heart failure defined as ejection fraction $<50 \%$. Area under the curve is $0.811, p<0.001$. (b) Receiver Operating Characteristic (ROC) curve of NT-proBNP with heart failure defined as ejection fraction $<40 \%$. Area under the curve is $0.902, p<0.001$.

NT-proBNP outperformed both sHLA-G and uric acid as a biomarker of LVSD (Figures 2-4).

There was no correlation between sHLA-G and uric acid, neither in the whole population nor in the patients with LVSD (Spearman, $p=0.295, n=256 ; p=0.529, n=105$ ).
There was a significant higher level of sHLA-G in cases of significant valvular heart disease for the whole study population of 260 subjects ( $p=0.002$, Mann-Whitney test Figure 5(a)). However, there was only a trend for the fraction of the population without LVSD ( $p=0.067$; Figure 5(b)). 


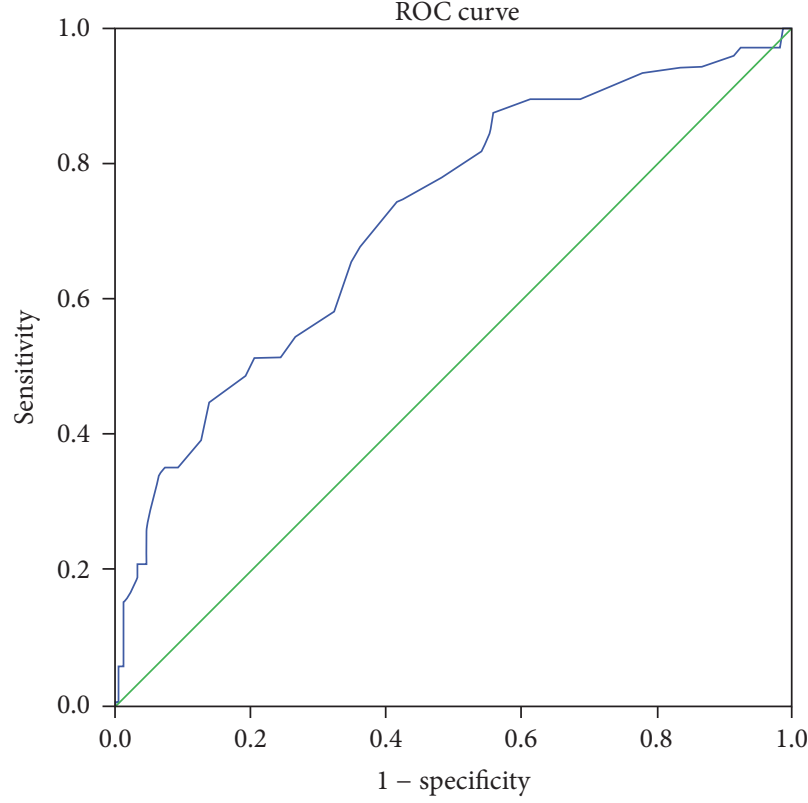

(a)

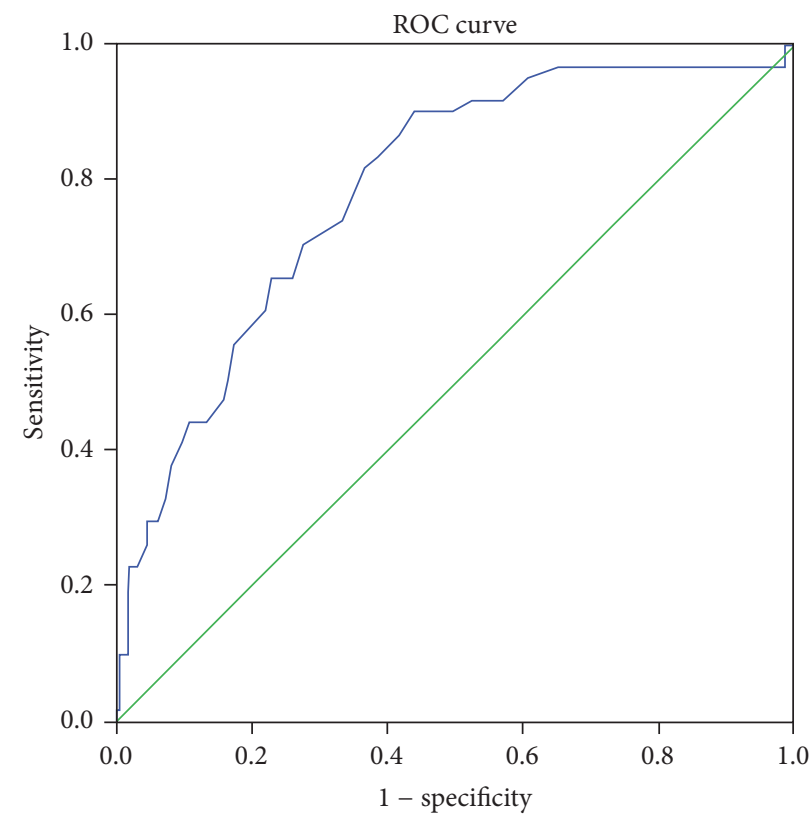

(b)

FIgURE 4: (a) Receiver Operating Characteristic (ROC) curve of uric acid with heart failure defined as ejection fraction $<50 \%$. Area under the curve is $0.721, p<0.001$. (b) Receiver Operating Characteristic (ROC) curve of uric acid with heart failure defined as ejection fraction $<40 \%$. Area under the curve is $0.788, p<0.001$.

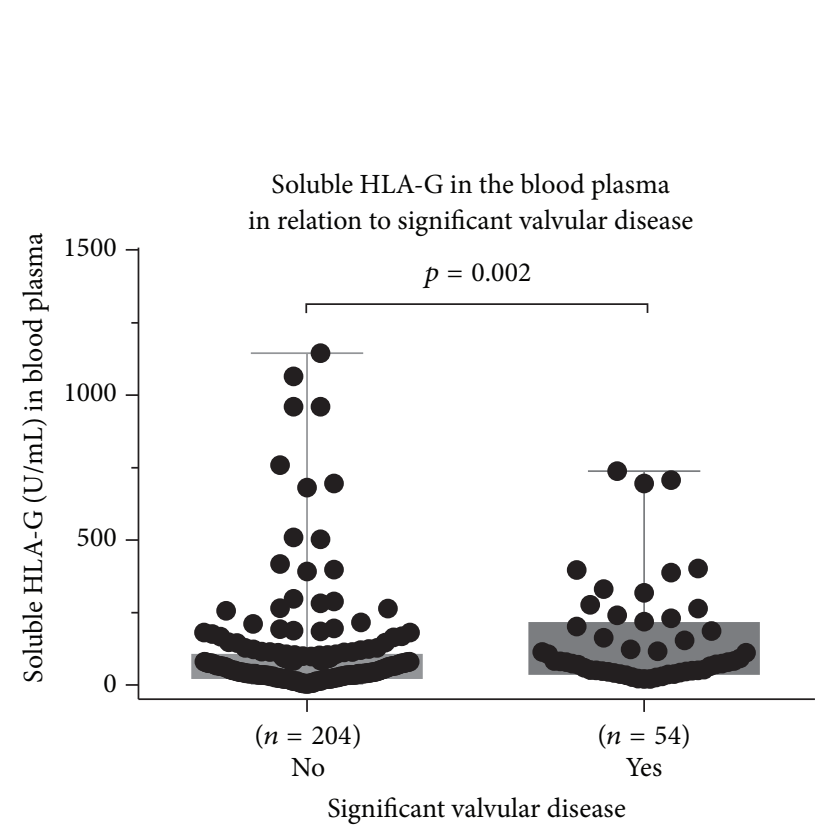

(a)

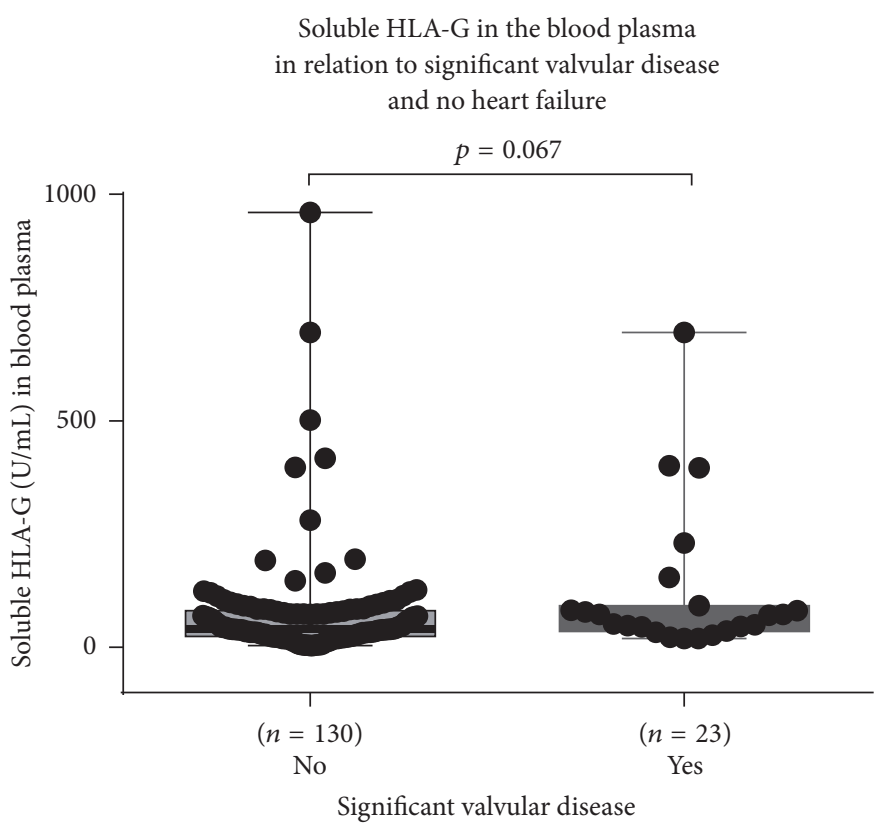

(b)

FIgURE 5: (a) Soluble HLA-G in peripheral blood in relation to significant valvular heart disease (box and whiskers plot, min. to max., all points shown; $p=0.002$, Mann-Whitney test). (b) Soluble HLA-G in peripheral blood in relation to significant valvular heart disease in patients without heart failure (EF $>50 \%)$ (box and whiskers plot, min. to max., all points shown; $p=0.067$, Mann-Whitney test).

3.3. HLA-G Gene Polymorphisms and Association with Risk of Heart Failure. There were no significant differences in the distributions of the $14 \mathrm{bp}$ ins/del genotypes between the different EF groups (Table 3; $p=0.82$, Chi-Square test). However, there was a tendency, when the group with $\mathrm{EF}>40 \%$ was compared with the group with $\mathrm{EF}<40 \%$ $(p=0.10$, Chi-Square test). In the group with $\mathrm{EF}>$ $40 \%$, the frequency of the Ins $14 \mathrm{bp} / \mathrm{Ins} 14 \mathrm{bp}$ genotype 
TABLE 3: Distribution of genotypes of the 14 bp insertion/deletion polymorphism in the $3^{\prime}$-untranslated region of the HLA-G gene in relation to left ventricular ejection fraction.

\begin{tabular}{|c|c|c|c|c|}
\hline Ejection fraction & Del 14 bp/Del 14 bp & Del 14 bp/Ins 14 bp & Ins 14 bp/Ins 14 bp & Total \\
\hline$>50 \%^{\mathrm{a}}$ & $61(39.6 \%)$ & $66(42.9 \%)$ & $27(17.5 \%)$ & $154(100.0 \%)$ \\
\hline $40 \%$ to $50 \%$ & $17(37.8 \%)$ & $17(37.8 \%)$ & $11(24.4 \%)$ & $45(100.0 \%)$ \\
\hline $30 \%$ to $40 \%$ & $12(40.0 \%)$ & $16(53.3 \%)$ & $2(6.7 \%)$ & $30(100.0 \%)$ \\
\hline$<30 \%$ & $12(38.7 \%)$ & $16(51.6 \%)$ & $3(9.7 \%)$ & $31(100.0 \%)$ \\
\hline$>40 \%^{\mathrm{b}}$ & $78(39.2 \%)$ & $83(41.7 \%)$ & $38(19.1 \%)$ & $199(100.0 \%)$ \\
\hline$<40 \%^{\mathrm{b}}$ & $24(39.3 \%)$ & $32(52.5 \%)$ & $5(8.2 \%)$ & $61(100.0 \%)$ \\
\hline
\end{tabular}

${ }^{\mathrm{a}} \mathrm{p}=0.82($ Chi-Square test, $0.40, \mathrm{df}=2) ;{ }^{\mathrm{b}} p=0.10$ (Chi-Square test, $\left.4.57, \mathrm{df}=2\right)$.

TABLE 4: Distribution of haplotypes of the 14 bp insertion/deletion polymorphism (Del/Ins) and the $+3142 \mathrm{SNP}(\mathrm{C} / \mathrm{G})$ in the $3^{\prime}$-untranslated region of the HLA-G gene in relation to left ventricular ejection fraction.

\begin{tabular}{lcccccccc}
\hline Ejection fraction & DelC/DelC & DelC/DelG & DelC/InsC & DelC/InsG & DelG/DelG & DelG/InsG & InsG/InsG & Total \\
\hline$>40 \%^{\mathrm{a}}$ & $62(31.2 \%)$ & $15(7.5 \%)$ & $1(0.5 \%)$ & $74(37.2 \%)$ & $1(0.5 \%)$ & $8(4.0 \%)$ & $38(19.1 \%)$ & $199(100.0 \%)$ \\
$<40 \%^{\mathrm{a}}$ & $20(32.8 \%)$ & $4(6.6 \%)$ & $0(0.0 \%)$ & $23(37.7 \%)$ & $0(0.0 \%)$ & $9(14.8 \%)$ & $5(8.2 \%)$ & $61(100.0 \%)$ \\
\hline
\end{tabular}

${ }^{\mathrm{a}} p=0.033$ (Chi-Square test, $10.50, \mathrm{df}=4$ ), for the Chi-Square test DelC/InsG was added to the DelC/DelC group and DelG/DelG was added to the DelG/InsG group.

was $19.1 \%$ and only $8.2 \%$ in the group with $\mathrm{EF}<40 \%$ (Table 3).

This difference was statistically significant, when the combined haplotype of the two HLA-G gene polymorphisms, the $14 \mathrm{bp}$ ins/del and the +3142 SNP, was analyzed (Table 4; $p=0.033$, Chi-Square test). The combined genotype of the haplotype InsG/InsG was more frequent among subjects with $\mathrm{EF}>40 \%$ than among patients with $\mathrm{EF}<40 \%$; the opposite was observed for the DelG/InsG combination of haplotypes.

In a separate analysis of the +3142 HLA-G SNP alone, no differences were observed in the distributions of the three genotypes between the group with $\mathrm{EF}<40 \%$ and the group with $\mathrm{EF}>40 \%$. For the group with $\mathrm{EF}>40 \%$, the frequencies were $\mathrm{C} / \mathrm{C}(32.2 \%), \mathrm{C} / \mathrm{G}(43.7 \%)$, and $\mathrm{G} / \mathrm{G}(24.1 \%)$. For the group with $\mathrm{EF}<40 \%$, they were C/C (32.8\%), C/G (44.3\%), and $\mathrm{G} / \mathrm{G}(23.0 \%)$.

\section{Discussion}

This study shows that sHLA-G is increased in patients with $\mathrm{EF}<40$ compared to patients with $\mathrm{EF} \geq 40 \%$. There was no difference in the concentration of sHLA-G in LVSD patients with $\mathrm{EF}<30 \%$ and with EF between 30 and $40 \%$. Thus, sHLA$\mathrm{G}$ in the blood plasma does not indicate the severity of LVSD, and, in accordance with the study by Almasood et al., we conclude that sHLA-G is a very sensitive LVSD biomarker $[8,9,24]$. The assay used in the current study detects both soluble HLA-G5 and soluble HLA-G1 associated with $\beta 2$ microglobulin. The source of sHLA-G in the blood from men and nonpregnant women is not well established but is probably derived from immune cells [21]. It can be speculated that the raise in sHLA-G associated with LVSD might originate from activated immune cells or from the heart.

Neither sHLA-G nor serum uric acid is comparable with NT-proBNP as LVSD-biomarker, primarily due to a poor specificity [5]. Soluble HLA-G is influenced by many other conditions, for example, cancer and autoimmune disease, which may confound the analysis and the results and reduce the suitability of sHLA-G as a biomarker for LVSD.

Specificity may be increased by genotyping for the combined haplotypes of the two tested HLA-G gene polymorphisms. An interesting novel finding in the current study was that the distribution of the combined haplotypes of the two tested HLA-G gene polymorphisms, the $14 \mathrm{bp}$ ins/del and the +3142 SNP in the $3^{\prime}$ UTR of the gene, was statistically significant between the subjects with EF $>40 \%$ and patients with $\mathrm{EF}<40 \%$ (Table 4; $p=0.033$ ). InsG/InsG was more frequent among subjects with $\mathrm{EF}>40 \%$ than patients with $\mathrm{EF}<40 \%$; the opposite was observed for DelG/InsG. Interestingly, in healthy blood donors, the Ins 14 bp/Ins 14 bp HLA-G genotype has been significantly associated with low sHLA-G levels in the blood in several studies [28, 29]. Furthermore, the DelG/InsG combination has also been associated with a higher level of sHLA-G than the InsG/InsG combination, for example, in patients with multiple sclerosis; however, the DelC/DelC combination showed the highest concentrations of sHLA-G in the same study [30]. This is supported by the observations in the current study, which could indicate that specific HLA-G gene polymorphisms or haplotypes might influence the sHLA-G level in the blood and thereby the individual sHLA-G response in specific patients with systolic heart failure. It is not known whether this is due to genetic or epigenetic factors or if it is an adaptive mechanism triggered by the inflammatory process $[19,20]$.

There are certain limitations to the present study. One limitation of the current study is that the number of study participants is rather small; however, it is still the largest study until now regarding HLA-G in subjects with and without systolic heart failure. The study reflects clinical practice. The diagnosis of heart failure is uncertain for the small group with EF in the grey zone of 40-50\%, but echocardiography is the standard diagnostic test and in this study performed by the most qualified $[1,2]$. Important confounding factors 
are morbidities other than systolic heart failure, and these are common and inevitable (Table 1).

One of the reactions of the body to injury is inflammation represented in this study by serum uric acid, which might trigger an immunologic response represented in this study by sHLA-G [10]. Both are biomarkers of LVSD, and thus inflammation and immune modulation seem to be involved in LVSD. This is in accordance with accumulating evidence that systemic and persistent inflammatory disorders predispose to cardiovascular diseases. This is the case in gout, rheumatoid arthritis, psoriasis, inflammatory bowel disease, lupus erythematosus, sclerosis disseminatus, and other autoimmune diseases and in chronic infections and cancers $[4,5,18,31]$. It is also observed in conditions associated with long-lasting low grade inflammation and endothelial dysfunctions like atherosclerosis, diabetes mellitus, the metabolic syndrome, venous thromboembolism, smoking, and affective disorders and in chronic heart failure $[4,12]$. Upregulation of HLA$\mathrm{G}$ is present in most of these disorders, which nevertheless are dominated by inflammation [18]. Out-of-balance inflammation with persistent rise in inflammatory cytokines seems to be the common denominator for many potentially coherent diseases and disorders, and it acts self-reinforcing in a complex vicious circle [4]. The inflammatory triggers and mediators are poorly understood, but they promote and regulate the inflammatory cascade that predisposes to, for example, atherosclerosis and LVSD [31].

HLA-G is elevated in many conditions, and thus there is a lack of diagnostic precision and specificity, which may obscure evaluation of the significance of HLA-G as a biomarker of LVSD in a multimorbid ageing population like the one in the current study. Furthermore, it cannot be determined from the current study, which role sHLA-G might have in the pathogenesis and the clinical course and prognosis of LVSD and whether it is a simple marker or participate in LVSD.

Likewise, the pathophysiologic effect of uric acid in LVSD is unknown. Serum uric acid has a negative correlation with $\mathrm{EF}[8,9]$. It is an independent risk factor for LVSD, but it is not known if it is cause, consequence, or simply an epiphenomenon. The serum uric acid concentration is increased in patients with chronic LVSD, probably due to both reduced renal excretion and augmented production $[8,9]$. Lowsodium diet, diuretics, and insulin resistance may increase reabsorption of uric acid. Cardiac and renal disorders are related and as cardiac function deteriorates with falling cardiac output, the glomerular filtration rate (GFR) falls, which leads to a reduction in renal uric acid excretion [10]. At the same time the inflammatory process associated with the chronic diseases accelerates, which contributes to an increase in serum uric acid, TNF, interleukin-1 and interleukin-6, and other cytokines and in sHLA-G [7]. The simultaneous elevation of uric acid and sHLA-G might represent different aspects of the same process, acting in negative feedback as proinflammatory and anti-inflammatory markers.

In LVSD there is an imbalance between proinflammatory and anti-inflammatory cytokines [32]. The clinical significance remains to be determined. There are indications that the prognosis may be improved by restraining the inflammatory process, and an increase in EF in LVSD has been observed following treatment with, for example, thalidomide, pentoxifylline, intravenous immunoglobulin, glucocorticoid, colchicines, methotrexate, biological agents, interleukin-10, influenza vaccination, HIV-therapy, antidiabetic sodium glucose cotransporters, and antidepressant drugs [4, 12, 32, 33]. However, no improvement was observed in a variety of antibiotic trials and in studies antagonizing TNF in patients with LVSD, and there have been mixed results in studies of the use of allopurinol, which inhibits xanthine oxidase $[4,8$, $11,12,31]$. In the future, therapies directed at downregulating or inhibiting inflammation may reduce atherosclerosis and its complications including heart failure [33]. Further studies are needed to elucidate the role of HLA-G in this scenario.

\section{Conclusion}

For the first time, it was shown in the current study that a combined haplotype (DelG/InsG) of two HLA-G gene polymorphisms, the $14 \mathrm{bp}$ ins/del and the +3142 SNP, were more frequent among patients with $\mathrm{EF}<40 \%$ than among subjects with $\mathrm{EF} \geq 40 \%$. The opposite was observed for the combined haplotype InsG/InsG. This probably influences the sHLA-G level in the blood, and this study also showed that sHLA-G was increased in patients with $\mathrm{EF}<40$ compared to patients with $\mathrm{EF} \geq 40 \%$.

\section{Competing Interests}

The authors declare that there were no financial or any other factors that may have led to a conflict of interests.

\section{Acknowledgments}

This work was supported by The Region Zealand Research Foundation of Cross-Sectional Health Projects. The authors would like to thank Anja S. Torbensen for helpful technical assistance regarding the measurements of sHLA-G and the HLA-G genotyping and Lili Hald Jensen for measurement of NT-proBNP. Furthermore, the authors would like to thank Roche Diagnostics for providing the diagnostic assays Elecsys 2010 system to measure NT-proBNP.

\section{References}

[1] P. Ponikowski, A. A. Voors, S. D. Anker et al., "2016 ESC Guidelines for the diagnosis and treatment of acute and chronic heart failure: the Task Force for the diagnosis and treatment of acute and chronic heart failure of the European Society of Cardiology (ESC) developed with the special contribution of the Heart Failure Association (HFA) of the ESC," European Heart Journal, vol. 37, no. 27, pp. 2129-2200, 2016.

[2] L. L. Olesen, A. Andersen, and S. Thaulow, "Hand-held echocardiography is useful for diagnosis of left systolic dysfunction in an elderly population," Danish Medical Journal, vol. 62, no. 7, Article ID A5100, pp. 1-5, 2015.

[3] A. Mosterd and A. W. Hoes, "Clinical epidemiology of heart failure," Heart, vol. 93, no. 9, pp. 1137-1146, 2007. 
[4] L. Gullestad, T. Ueland, L. E. Vinge, A. Finsen, A. Yndestad, and P. Aukrust, "Inflammatory cytokines in heart failure: mediators and markers," Cardiology, vol. 122, no. 1, pp. 23-35, 2012.

[5] A. Maisel, C. Mueller, K. Adams Jr. et al., "State of the art: using natriuretic peptide levels in clinical practice," European Journal of Heart Failure, vol. 10, no. 9, pp. 824-839, 2008.

[6] L. L. Olesen and A. Andersen, "ECG as a first step in the detection of left ventricular systolic dysfunction in the elderly," ESC Heart Failure, vol. 3, no. 1, pp. 44-52, 2016.

[7] C. Ruggiero, A. Cherubini, A. Ble et al., "Uric acid and inflammatory markers," European Heart Journal, vol. 27, no. 10, pp. 1174-1181, 2006.

[8] G. S. Filippatos, M. I. Ahmed, J. D. Gladden et al., "Hyperuricaemia, chronic kidney disease, and outcomes in heart failure: potential mechanistic insights from epidemiological data," European Heart Journal, vol. 32, no. 6, pp. 712-720, 2011.

[9] G. Lippi, M. Montagnana, M. Franchini, E. J. Favaloro, and G. Targher, "The paradoxical relationship between serum uric acid and cardiovascular disease," Clinica Chimica Acta, vol. 392, no. 1-2, pp. 1-7, 2008.

[10] F. Leyva, S. D. Anker, I. F. Godsland et al., "Uric acid in chronic heart failure: a marker of chronic inflammation," European Heart Journal, vol. 19, no. 12, pp. 1814-1822, 1998.

[11] D. L. Mann, "Innate immunity and the failing heart. The cytokine hypothesis revisited," Circulation Research, vol. 116, no. 7, pp. 1254-1268, 2015.

[12] E. Oikonomou, D. Tousoulis, G. Siasos, M. Zaromitidou, A. G. Papavassiliou, and C. Stefanadis, "The role of inflammation in heart failure: new therapeutic approaches," Hellenic Journal of Cardiology, vol. 52, no. 1, pp. 30-40, 2011.

[13] T. V. F. Hviid, "HLA-G in human reproduction: aspects of genetics, function and pregnancy complications," Human Reproduction Update, vol. 12, no. 3, pp. 209-232, 2006.

[14] M. H. Larsen and T. V. F. Hviid, "Human leukocyte antigen-G polymorphism in relation to expression, function, and disease," Human Immunology, vol. 70, no. 12, pp. 1026-1034, 2009.

[15] E. A. Donadi, E. C. Castelli, A. Arnaiz-Villena, M. Roger, D. Rey, and P. Moreau, "Implications of the polymorphism of HLA-G on its function, regulation, evolution and disease association," Cellular and Molecular Life Sciences, vol. 68, no. 3, pp. 369-395, 2011.

[16] E. D. Carosella, N. Rouas-Freiss, D. T.-L. Roux, P. Moreau, and J. LeMaoult, "HLA-G. an immune checkpoint molecule," Advances in Immunology, vol. 127, pp. 33-144, 2015.

[17] R. Sheshgiri, N. Rouas-Freiss, V. Rao et al., "Myocardial HLA$\mathrm{G}$ reliably indicates a low risk of acute cellular rejection in heart transplant recipients," The Journal of Heart and Lung Transplantation, vol. 27, no. 5, pp. 522-527, 2008.

[18] N. Rouas-Freiss, P. Moreau, J. LeMaoult, and E. D. Carosella, "The dual role of HLA-G in cancer," Journal of Immunology Research, vol. 2014, Article ID 359748, 10 pages, 2014.

[19] L. L. Nilsson, S. Djurisic, and T. V. F. Hviid, "Controlling the immunological crosstalk during conception and pregnancy: HLA-G in reproduction," Frontiers in Immunology, vol. 5, article 198, 2014.

[20] S. Djurisic and T. V. F. Hviid, "HLA class Ib molecules and immune cells in pregnancy and preeclampsia," Frontiers in Immunology, vol. 5, article 652, 2014.

[21] V. Rebmann, J. Regel, D. Stolke, and H. Grosse-Wilde, "Secretion of sHLA-G molecules in malignancies," Seminars in Cancer Biology, vol. 13, no. 5, pp. 371-377, 2003.
[22] A. Ishitani and D. E. Geraghty, "Alternative splicing of HLA-G transcripts yields proteins with primary structures resembling both class I and class II antigens," Proceedings of the National Academy of Sciences of the United States of America, vol. 89, no. 9, pp. 3947-3951, 1992.

[23] G. M. Park, S. Lee, B. Park et al., "Soluble HLA-G generated by proteolytic shedding inhibits NK-mediated cell lysis," Biochemical and Biophysical Research Communications, vol. 313, no. 3, pp. 606-611, 2004.

[24] A. Almasood, R. Sheshgiri, J. M. Joseph et al., "Human leukocyte antigen- $\mathrm{G}$ is upregulated in heart failure patients: a potential novel biomarker,' Human Immunology, vol. 72, no. 11, pp. 1064-1067, 2011.

[25] S. Djurisic, A. E. Sørensen, and T. V. F. Hviid, "A fast and easy real-time PCR genotyping method for the HLA-G 14-bp insertion/deletion polymorphism in the $3^{\prime}$ untranslated region," Tissue Antigens, vol. 79, no. 3, pp. 186-189, 2012.

[26] D. Bortolotti, V. Gentili, L. Melchiorri, A. Rotola, and R. Rizzo, "An accurate and reliable real time SNP genotyping assay for the HLA-G +3142 bp C > G polymorphism," Tissue Antigens, vol. 80, pp. 259-262, 2012.

[27] W. H. Hall, R. Ramachandran, S. Narayan, A. B. Jani, and S. Vijayakumar, "An electronic application for rapidly calculating Charlson comorbidity score," BMC Cancer, vol. 4, article 94, pp. $1-8,2004$.

[28] T. V. F. Hviid, R. Rizzo, O. B. Christiansen, L. Melchiorri, A. Lindhard, and O. R. Baricordi, "HLA-G and IL-10 in serum in relation to HLA-G genotype and polymorphisms," Immunogenetics, vol. 56, no. 3, pp. 135-141, 2004.

[29] X.-Y. Chen, W.-H. Yan, A. Lin, H.-H. Xu, J.-G. Zhang, and X.$\mathrm{X}$. Wang, "The 14 bp deletion polymorphisms in HLA-G gene play an important role in the expression of soluble HLA-G in plasma," Tissue Antigens, vol. 72, no. 4, pp. 335-341, 2008.

[30] R. Rizzo, D. Bortolotti, N. Ben Fredj et al., "Role of HLA$\mathrm{G} 14 \mathrm{bp}$ deletion/insertion and $+3142 \mathrm{C}>\mathrm{G}$ polymorphisms in the production of sHLA-G molecules in relapsing-remitting multiple sclerosis," Human Immunology, vol. 73, no. 11, pp. 11401146, 2012.

[31] J. S. Zebrack and J. L. Anderson, "The role of inflammation and infection in the pathogenesis and evolution of coronary artery disease," Current Cardiology Reports, vol. 4, no. 4, pp. 278-288, 2002.

[32] C. Stumpf, K. Seybold, S. Petzi et al., "Interleukin-10 improves left ventricular function in rats with heart failure subsequent to myocardial infarction," European Journal of Heart Failure, vol. 10, no. 8, pp. 733-739, 2008.

[33] P. M. Ridker and T. F. Lüscher, "Anti-inflammatory therapies for cardiovascular disease," European Heart Journal, vol. 35, no. 27, pp. 1782-1791, 2014. 


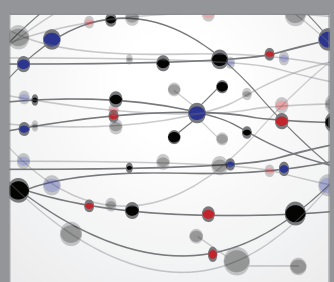

The Scientific World Journal
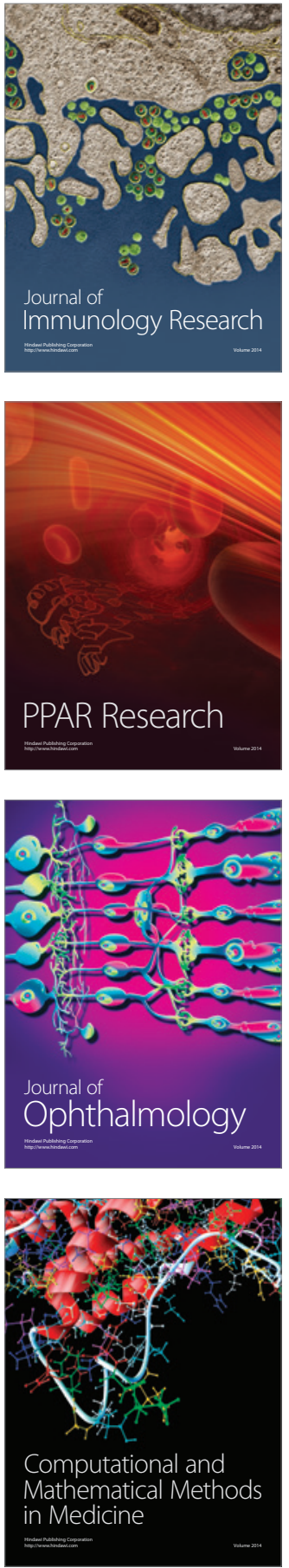

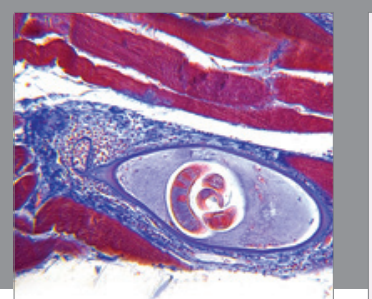

Gastroenterology Research and Practice

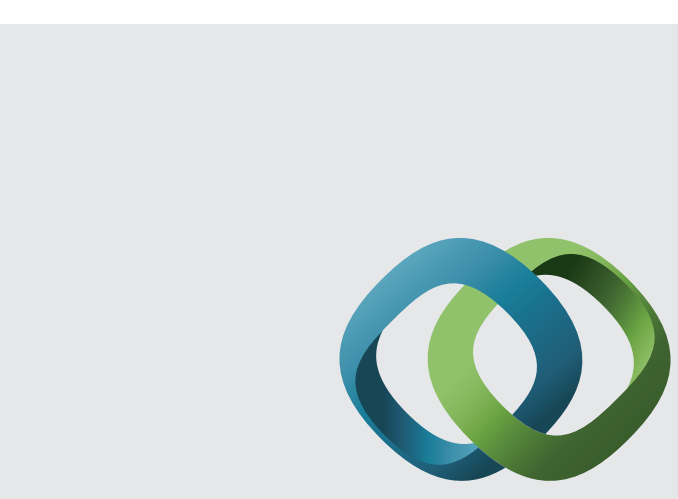

\section{Hindawi}

Submit your manuscripts at

http://www.hindawi.com
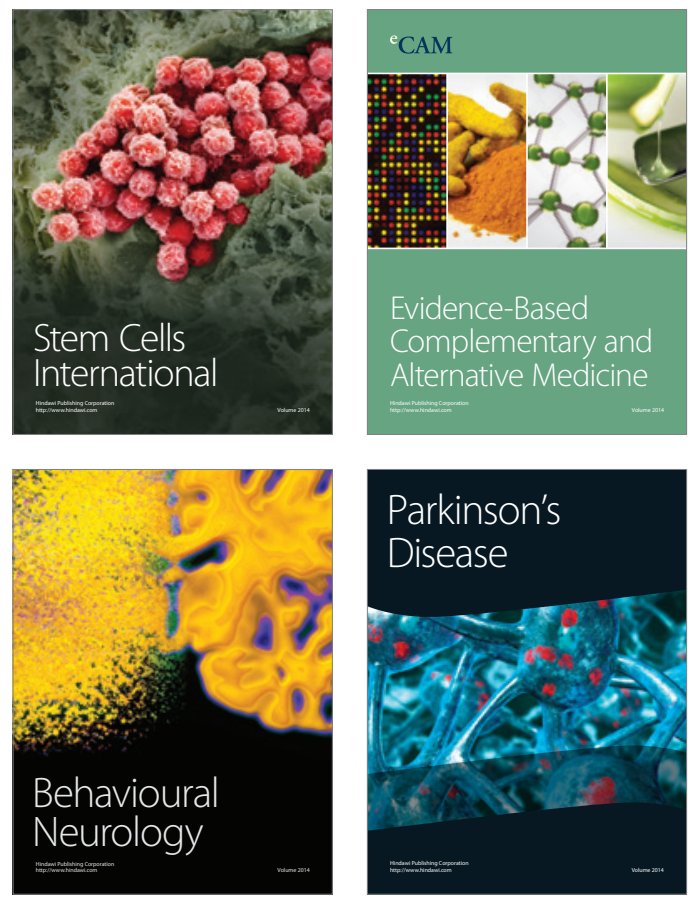
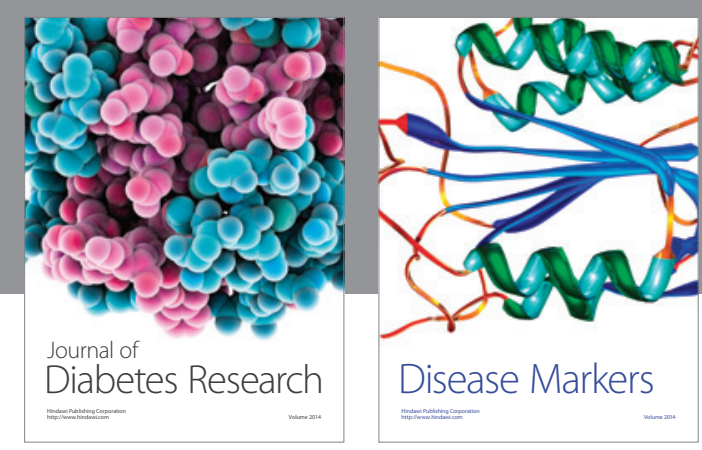

Disease Markers
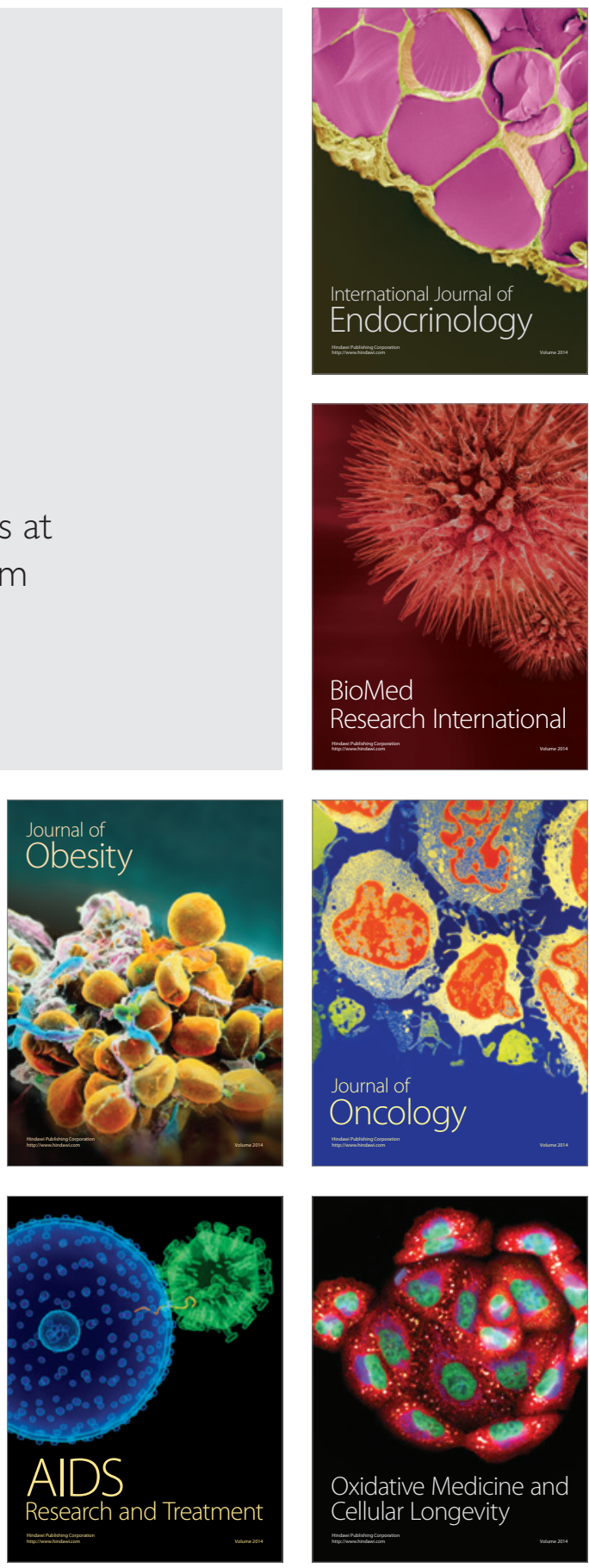